\title{
Subpectoral versus suprapectoral biceps tenodesis yields similar clinical outcomes: a systematic review
}

\author{
John W Belk, ${ }^{1}$ Stephen G Thon, ${ }^{1,2}$ John Hart, ${ }^{1,2}$ Eric C McCarty, Jr., ${ }^{1}$ Eric C McCarty ${ }^{1,2}$
}

${ }^{1}$ Department of Orthopaedics, University of Colorado at Boulder, Boulder, Colorado, USA ${ }^{2}$ Department of Orthopedics, University of Colorado Denver School of Medicine, Aurora, Colorado, USA

\section{Correspondence to}

Dr Eric C McCarty, Orthopaedics, University of Colorado at Boulder, Boulder, CO 80309, USA;

eric.mccarty@cuanschutz.edu

Received 29 September 2020 Revised 13 April 2021 Accepted 16 April 2021 Published Online First 20 May 2021

\section{Check for updates}

(C) International Society of Arthroscopy, Knee Surgery and Orthopaedic Sports Medicine 2021. No commercial re-use. See rights and permissions. Published by BMJ.

To cite: Belk JW, Thon SG, Hart J, et al. J ISAKOS 2021:6:356-362.

\section{ABSTRACT}

Importance Arthroscopic suprapectoral biceps tenodesis (ABT) and open subpectoral biceps tenodesis (OBT) are two surgical treatment options for relief of long head of the biceps tendon (LHBT) pathology and superior labrum anterior to posterior (SLAP) tears. There is insufficient knowledge regarding the clinical superiority of one technique over the other.

Objective To systematically review the literature in order to compare the clinical outcomes and safety of $A B T$ and OBT for treatment of LHBT or SLAP pathology.

Evidence review A systematic review was performed by searching PubMed, the Cochrane Library and Embase to identify studies that compared the clinical efficacy of $A B T$ versus OBT. The search phrase used was: (bicep $O R$ biceps OR biceps brachii OR long head of biceps brachii OR biceps tendinopathy) AND (tenodesis). Patients were assessed based on the American Shoulder and Elbow Surgeons Score, the visual analogue scale, the Single Assessment Numeric Evaluation, Constant-Murley Score, clinical failure, range of motion, bicipital groove pain and strength. Preferred Reporting Items for Systematic Reviews and Meta-Analyses guidelines were followed, and both the Cochrane Collaboration's and Risk of Bias in Non-randomised Studies - of Interventions (ROBINS-I) risk of bias tools were used to evaluate risk of bias.

Findings Eight studies (one level I, seven level III) met inclusion criteria, including 326 patients undergoing $A B T$ and 381 patients undergoing OBT. No differences were found in treatment failure rates or patient-reported outcome scores between groups in any study. One study found OBT patients to experience significantly increased range of shoulder forward flexion when compared with ABT patients $(p=0.049)$. Two studies found ABT patients to experience significantly more postoperative stiffness when compared with OBT patients $(p<0.05)$.

Conclusions Patients undergoing ABT and OBT can be expected to experience similar improvements in clinical outcomes at latest follow-up without differences treatment failure or functional performance. ABT patients may experience an increased incidence of stiffness in the early postoperative period.

Level of evidence III.

\section{INTRODUCTION}

Direct disruption or tendinopathy of the proximal long head of the biceps tendon (LHBT) along with indirect disruption through superior labrum anterior to posterior (SLAP) lesions or biceps pulley lesions is a known source of anterior shoulder pain. ${ }^{1}$ LHBT pathology is often caused by tendon degeneration resulting from persistent inflammation or microtearing, tendon anchor disorders (SLAP lesions) and LHBT instability. ${ }^{2}$ Though commonly seen in association with other

\section{What is already known}

Arthroscopic suprapectoral biceps tenodesis and open subpectoral biceps tenodesis are two treatment options for long head of the biceps tendon and superior labrum anterior to posterior pathology.

- The superiority of one technique over the other and their comparative effects on clinical outcomes and postoperative patient functionality remains unclear.

\section{What are the new findings}

Arthroscopic suprapectoral biceps tenodesis patients may experience an increased incidence in stiffness in the early postoperative period.

- Tenodesis technique does not significantly affect treatment failure rates or functional performance.

shoulder pathology, especially rotator cuff tears ${ }^{3}$ and glenohumeral joint osteoarthritis, ${ }^{4}$ injury to the biceps tendon is recognised as a significant, independent source of pain if left untreated. ${ }^{5}$ Following failure of conservative management, arthroscopic suprapectoral biceps tenodesis (ABT) and open subpectoral biceps tenodesis (OBT) are two of the most common procedures to treat LHBT pathology. ${ }^{6}$ While OBT has been demonstrated to be associated with higher complication rates, which is likely due to the increased invasiveness of this procedure, ${ }^{7}$ it has also been shown to reduce stiffness and bicipital groove pain in the early postoperative period. ${ }^{8-11}$ While both techniques have been demonstrated to produce favourable clinical outcomes, ${ }^{12}$ the superiority of one technique over the other remains unclear, and there is limited information regarding the postoperative complication rates and clinical functionality following these two tenodesis techniques. The purpose of this systematic review was to compare the clinical outcomes and safety of ABT and OBT for the treatment of LHBT or SLAP pathology during shoulder arthroscopy.

\section{METHODS}

This systematic review was conducted according to Preferred Reporting Items for Systematic Reviews and Meta-Analyses (PRISMA) guidelines using a PRISMA checklist. Two independent reviewers (JWB and ST) searched the PubMed, Embase and Cochrane Library databases up to 25 May 2020. The electronic search strategy used was: (bicep OR biceps OR biceps brachii OR long head of biceps brachii OR biceps tendinopathy) 
AND (tenodesis). A total of 1263 studies were reviewed by title and/ or abstract to determine study eligibility based on inclusion criteria. In cases of disagreement, a third reviewer (ECM) made the final decision.

The inclusion criteria were non-overlapping human studies that directly compared clinical and functional outcomes of patients undergoing suprapectoral vs subpectoral biceps tenodesis, studies that were published in English and studies published in a peer-reviewed journal. Exclusion criteria included nonhuman studies, studies that focused on procedures other than suprapectoral and subpectoral biceps tenodesis, case series, review studies, cadaveric studies and biomechanical studies. Data extraction from each study was performed independently and then reviewed by a second author (ST). There was no need for funding or a third party to obtain any of the collected data.

Risk of bias was assessed according to the Cochrane Collaboration's risk of bias tool ${ }^{13}$ for one randomised study, ${ }^{14}$ which incorporates an assessment of randomisation, blinding, completeness of outcomes data, selection of outcomes reported and other sources of bias. For the remaining seven, non-randomised studies, ${ }^{7-111516}$ risk of bias was assessed according to the Risk of Bias in Non-Randomized Studies - of Interventions (ROBINS-I) risk of bias tool, ${ }^{17}$ which incorporates an assessment of bias due to confounding, selection of participants, deviations from intended interventions, completeness of outcomes data, selection of outcomes reported and other sources of bias. A Cohen's kappa score was calculated to determine the level of intraobserver agreement between reviewers. A score of $<0.20$ indicates poor agreement; $0.21-0.40$ as fair agreement; $0.41-0.60$ as moderate agreement; $0.61-0.80$ as good agreement; and 0.81-1.00 as very good agreement. ${ }^{18}$

\section{Reporting outcomes}

Outcomes assessed included patient-reported outcomes (PROs), postoperative functional evaluations and clinical failure. PROs included the American Shoulder and Elbow Surgeons (ASES) Score ${ }^{19}$ the visual analogue scale (VAS) for pain, the ConstantMurley Score ${ }^{20}$ and the Single Assessment Numeric Evaluation (SANE) Score. ${ }^{21}$ Postoperative functional evaluations included range of motion (ROM), bicipital groove pain, cosmetic deformity and strength. All studies ${ }^{7-11} 1^{14-16}$ used the ASES Score, four studies ${ }^{8101116}$ used the VAS Score, four studies ${ }^{81115}$ used the Constant-Murley Score and three studies ${ }^{914}{ }^{15}$ used the SANE Score. All studies ${ }^{7-11}$ 14-16 assessed postoperative ROM and bicipital groove pain, and five studies ${ }^{79}$ 14-16 reported on postoperative strength. Four studies ${ }^{7} 1115$ reported on cosmetic deformities postoperatively, and seven studies ${ }^{7-11} 1516$ reported on clinical failure. One cohort ${ }^{9}$ included outcomes that were published on separate occasions, ${ }^{922}$ though this was treated as one study for the sake of this review.

\section{Study methodology assessment}

The Modified Coleman Methodology Score (MCMS) ${ }^{23}$ was used to evaluate study methodology quality. The MCMS has a scaled potential score ranging from 0 to 100 . Scores ranging from 85 to 100 are excellent, 70-84 are good, 55-69 are fair and less than 55 are poor.

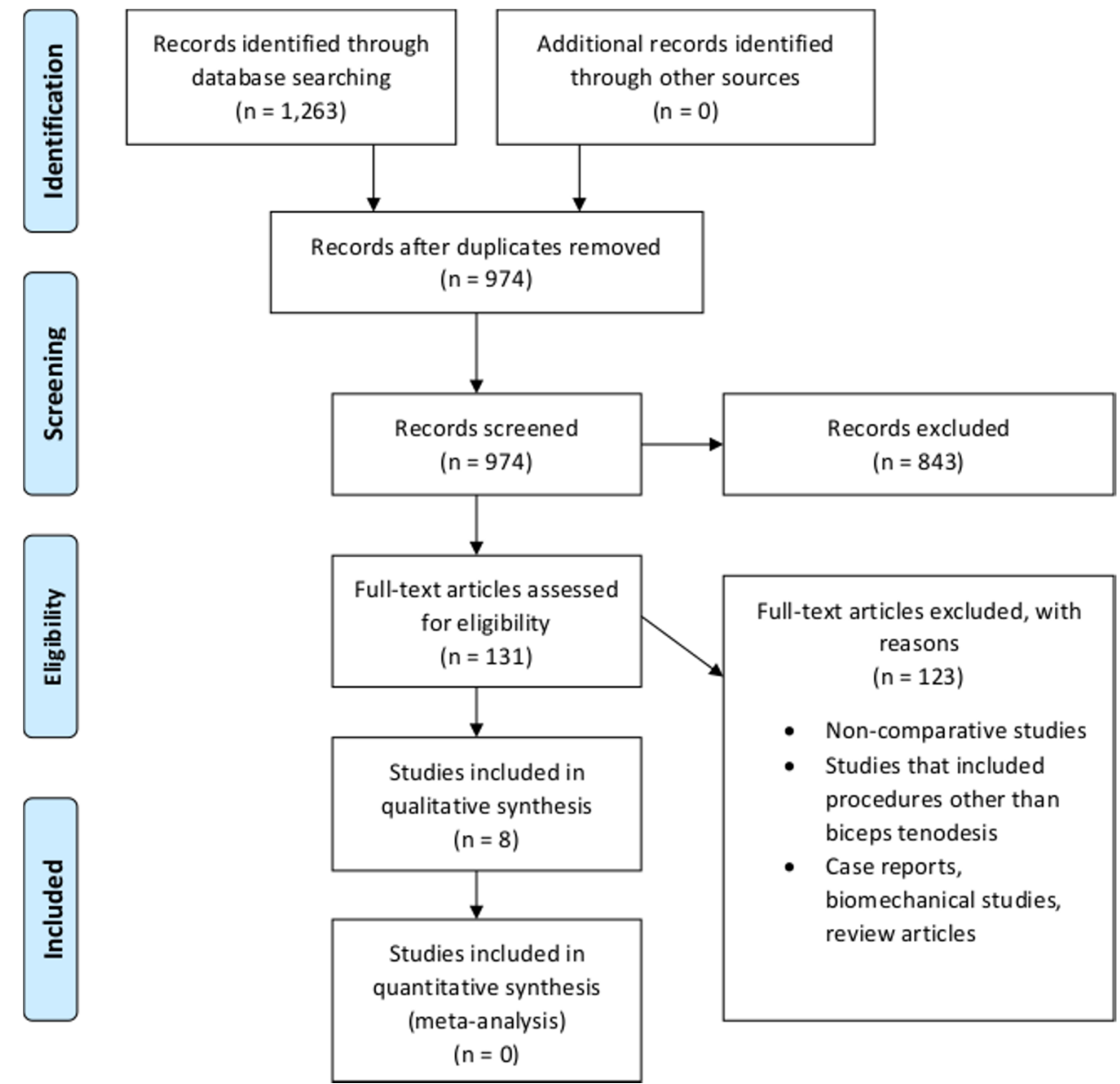

Figure 1 Preferred Reporting Items for Systematic Reviews and Meta-Analyses flow diagram. 
Table 1 Studies included

\begin{tabular}{|c|c|c|c|c|c|c|}
\hline Study & $\begin{array}{l}\mathrm{N} \\
\text { (ABT, OBT) }\end{array}$ & Level of evidence & $\begin{array}{l}\text { Patient age, years } \\
\text { (ABT) }\end{array}$ & $\begin{array}{l}\text { Patient age, years } \\
\text { (OBT) }\end{array}$ & Follow-up, months & $\begin{array}{l}\text { Sex, \% male } \\
\text { (ABT, OBT) }\end{array}$ \\
\hline Duchman et al ${ }^{15}$ & 20,25 & III & $49.9 \pm 11.8$ & $38.9 \pm 11.0$ & $38.4 \pm 13.2$ & $75.0,88.0$ \\
\hline Forsythe et al ${ }^{14}$ & 37,38 & I & $50.2 \pm 10.5$ & $50.3 \pm 10.4$ & $12.6 \pm 0.8$ & $73.0,60.5$ \\
\hline Gombera et al ${ }^{7}$ & 23,23 & III & $57.3 \pm 6.8$ & $56.9 \pm 6.7$ & $30.2 \pm 6.8$ & NR \\
\hline Green et $a l^{16}$ & 15,23 & III & $60.0 \pm 10.2$ & $56.6 \pm 10.7$ & $50.2 \pm 15.6$ & $66.7,91.3$ \\
\hline Tu et $a l^{8}$ & 55,62 & III & $55.1 \pm 8.7$ & $57.4 \pm 8.8$ & $20.3 \pm 7.3$ & $47.3,46.8$ \\
\hline Werner et $a l^{9 *}$ & 106,143 & III & $51.5 \pm 9.5$ & $53.5 \pm 11.2$ & $9.7 \pm 2.3$ & $61.3,72.0$ \\
\hline Yi et $a l^{11}$ & 34,32 & III & $55.8 \pm 8.4$ & $54.4 \pm 7.9$ & 26.8 (median) & $41.2,46.9$ \\
\hline Yi et $a l^{10}$ & 36,35 & III & $54.3 \pm 10.7$ & $53.5 \pm 8.5$ & $21.2 \pm 1.7$ & $48.6,44.4$ \\
\hline
\end{tabular}

Patient age and follow-up are reported as mean (range).

*Outcomes from this cohort were published in two different articles, ${ }^{922}$ which were considered as one study in this systematic review.

$A B T$, arthroscopic suprapectoral biceps tenodesis; NR, not reported; OBT, open subpectoral biceps tenodesis.

\section{Statistical analysis}

A Cohen's kappa score was calculated to determine the level of intraobserver agreement between reviewers, with a 95\% CI reported with the final score.

\section{RESULTS}

Eight studies $^{7-11}{ }^{14-16}$ met inclusion and exclusion criteria (figure 1), including a total of 707 patients (ABT 326, OBT 381). One cohort ${ }^{9}$ included outcomes that were published on separate occasions, ${ }^{922}$ though this was treated as one study for the sake of this review. The study ${ }^{9}$ with the majority of outcomes available was considered as the 'included' study (table 1).

\section{Surgical technique}

\section{Arthroscopic suprapectoral biceps tenodesis}

All studies ${ }^{71114-16}$ described performing ABT in similar fashions. Patients were placed in either the beach chair ${ }^{7-9} 1114$ or lateral decubitus position. ${ }^{16}$ Two studies ${ }^{10} 15$ did not describe patient position. After a diagnostic arthroscopy, the biceps tendon was tagged using either a \#2 braided suture ${ }^{7-11} 1516$ or Krackow whipstitches, ${ }^{14}$ and excision of the proximal biceps tendon was performed at the biceps-labral junction. Anterolateral and posterolateral portals were then established, and the transverse ligaments overlying the biceps tendon within the bicipital groove were visualised. All studies ${ }^{7-11} 14-16$ described using a subdeltoid bursectomy to help with identification of the tendon space. All but one study ${ }^{11}$ described excising $15-20 \mathrm{~mm}$ of the most proximal aspect of the tendon, followed by careful placement of a locking \#2 braided suture. A unicortical bone tunnel was then drilled in the bicipital groove using a guidewire to help with precise placement. Finally, the tendon was fixed into the proximal humerus with $6.5-8.0 \mathrm{~mm}$ diameter bioabsorbable tenodesis screws, ${ }^{14-16}$ a size 8 Biceptor (Smith \& Nephew) interference screw $^{79}$ or a Swivelock screw (Arthrex GmbH). ${ }^{8}{ }^{911}$ To provide additional fixation strength, the suture tails are tied to one another using an arthroscopic knot pusher.

\section{Open subpectoral biceps tenodesis}

All studies ${ }^{7-11}{ }^{14-16}$ described performing OBT in similar fashions. Patients were placed in either the beach chair ${ }^{7-9} 1114$ or lateral decubitus position. ${ }^{16}$ Two studies ${ }^{10} 15$ did not describe patient position. After a diagnostic arthroscopy, a $2-4 \mathrm{~cm}$ incision was then made in the axilla centred over the inferior border of the pectoralis major tendon. The biceps tendon was externalised, and the diseased portion of the tendon was amputated. One study ${ }^{9}$ described performing excision of the diseased tendon arthroscopically. Following identification, the biceps tendon was delivered from the wound, and either \#2 braided sutures ${ }^{7-11} 1516$ or Krakow whipstitches ${ }^{14}$ were placed $15-20 \mathrm{~mm}$ proximal to the myotendinous junction. Care was taken while placing the medial retractor so as to protect the musculocutaneous nerve and avoid injury to the brachial plexus. A unicortical bone tunnel was then drilled in the bicipital groove using a guidewire to help with precise placement. Finally, the tendon was fixed into the proximal humerus with $6.5-8.0 \mathrm{~mm}$ diameter bioabsorbable tenodesis screws, ${ }^{14-16}$ a size 8 Biceptor (Smith \& Nephew) interference screw ${ }^{79}$ or a Swivelock screw (Arthrex GmbH). ${ }^{811}$ The tag ends of the suture are then tied to reinforce the repair and cut to complete the procedure.

\section{Concomitant procedures}

Multiple studies included patients undergoing concomitant procedures, including rotator cuff repair (RCR), ${ }^{8-11} 1415$ labral repair, ${ }^{7-9} 1415$ glenohumeral debridement, ${ }^{14}{ }^{15}$ subacromial decompression $^{7914} 15$ or distal clavicle excision. ${ }^{914} 15$ One study ${ }^{16}$ excluded patients undergoing procedures in addition to biceps tenodesis, and one study ${ }^{11}$ did not report the inclusion or exclusion of patients undergoing concomitant procedures. One study ${ }^{15}$ reported a significant difference in the percentage of patients undergoing concomitant RCR, in which a significantly higher number of ABT patients underwent RCR $(p=0.03)$.

\section{Modified Coleman Methodology Score}

Table 2 shows the MCMS scores from the eight included studies. Four studies ${ }^{71114}$ received good scores and four studies 9101516 received fair scores.

\section{Demographics}

One study ${ }^{15}$ reported a significant difference in age between the ABT and OBT, in which OBT patients were significantly younger $(p=0.003)$. Multiple studies reported no differences in sex, ${ }^{7-11} 1415$ age, ${ }^{7-1114}$ body mass index ${ }^{8-101416}$ or smoking status. $^{8} 10111516$

\section{Methodological quality assessment}

The results of the methodological quality assessment of the seven non-randomised studies using the ROBINS-I risk of bias tool are presented in figure 2. All eight studies ${ }^{7-11} 1^{14-16}$ showed a moderate risk of bias due to confounding, as there were no prognostic variables that predicted baseline intervention and no patients that switched between interventions during the study period. No studies excluded eligible patients or used variable follow-up times based on intervention (low risk of bias), 


\begin{tabular}{|c|c|}
\hline Study & MCMS \\
\hline Duchman et al ${ }^{15}$ & 68 \\
\hline Forsythe et $a l^{14}$ & 76 \\
\hline Gombera et al ${ }^{7}$ & 70 \\
\hline Green et al ${ }^{16}$ & 64 \\
\hline Tu et $a l^{8}$ & 75 \\
\hline Werner et $a l^{9 *}$ & 69 \\
\hline Yi et al ${ }^{11}$ & 73 \\
\hline Yi et $a l^{10}$ & 64 \\
\hline
\end{tabular}

except in one study ${ }^{16}$ in which the ABT group had a significantly decreased mean follow-up duration compared with the OBT group (serious risk of bias). No studies deviated from the intended intervention (low risk of bias), and all studies clearly classified treatment type (low risk of bias). All studies ${ }^{7-11}$ 14-16 used non-blinded outcome assessors by nature due to the presence or absence of an axillary incision but reported using identical postoperative protocols between groups (moderate risk of bias). Physicians were not blinded to treatment group in any study due to the nature of treatment (moderate risk of bias). No studies showed bias due to missing data (low risk of bias). Finally, no studies showed bias due to selective reporting (low risk of bias). A Cohen's kappa score of 0.82 (95\% CI 0.71 to 0.93 ) reflected a very good agreement between reviewers.

The one randomised study ${ }^{14}$ was assessed for methodological quality using the Cochrane Collaboration's risk of bias tool. ${ }^{13}$ Sequence generation and allocation were adequately reported (low risk of bias), though the study was deemed to be at moderate risk of detection bias due to unclear blinding of the outcome assessor. Patients were aware of their assigned intervention group due to the presence or absence of an axillary incision depending on the which surgery they underwent (high risk of bias). This study was not deemed to be at risk of bias for significant loss of follow-up, selective reporting or incomplete outcome data (low risk of bias).

\begin{tabular}{lllll} 
Table 3 & Treatment failure rates & & & \\
\hline Study & ABT & OBT & Total & P value \\
\hline Duchman et al $l^{15}$ & $1 / 20(5.0)$ & $0 / 25(0)$ & $1 / 45(10.3)$ & 0.26 \\
${\text { Gombera et } a l^{7}}_{\text {Green et } a l^{16}}^{0 / 23(0)}$ & $0 / 23(0)$ & $0 / 46(0)$ & 0.99 \\
Tu et $a l^{8}$ & $0 / 15(0)$ & $0 / 23(0)$ & $0 / 38(0)$ & 0.99 \\
Werner et al $^{9 *}$ & $0 / 55(0)$ & $0 / 62(0)$ & $0 / 117(0)$ & 0.99 \\
Yi et al & $0 / 27(0)$ & $0 / 35(0)$ & $0 / 62(0)$ & 0.99 \\
Yi et al $^{10}$ & $1 / 34(2.9)$ & $0 / 32(0)$ & $1 / 66(1.5)$ & 0.31 \\
\hline
\end{tabular}

Failures are reported as number of failures/total number of patients (\%) at latest follow-up.

* Only 62 patients from the Werner et $a l^{9}$ cohort were included at latest follow-up. ABT, arthroscopic suprapectoral biceps tenodesis; OBT, open subpectoral biceps tenodesis.

\section{Clinical failure}

Seven studies ${ }^{7-11} 1516$ defined treatment failure as the failure of proximal fixation of the repaired biceps tendon, usually visualised via ultrasound, MRI or the presence of a 'popeye' deformity. There were no significant differences between groups in any individual study (table 3 ).

\section{Bicipital groove pain and cosmetic deformities}

Six studies ${ }^{7-10} 1415$ defined bicipital groove pain as tenderness with palpation of the bicipital groove through the subpectoral triangle, Speed's test or Yergason's supination test. One study ${ }^{9}$ defined bicipital groove pain as postoperative stiffness. There were no significant differences between groups in any individual study (table 4).

Four studies ${ }^{781115}$ reported on the development of 'popeye' deformities at latest follow-up. From all four studies, there was only one patient in each group (one ABT and one OBT) who suffered from a popeye deformity.

Patient-reported outcomes

All studies ${ }^{7-11} 1416$ reported using the ASES Score, four studies $^{81011} 16$ reported using the VAS, four studies ${ }^{8} 91115$ reported using the Constant-Murley Scores and three studies ${ }^{91415}$

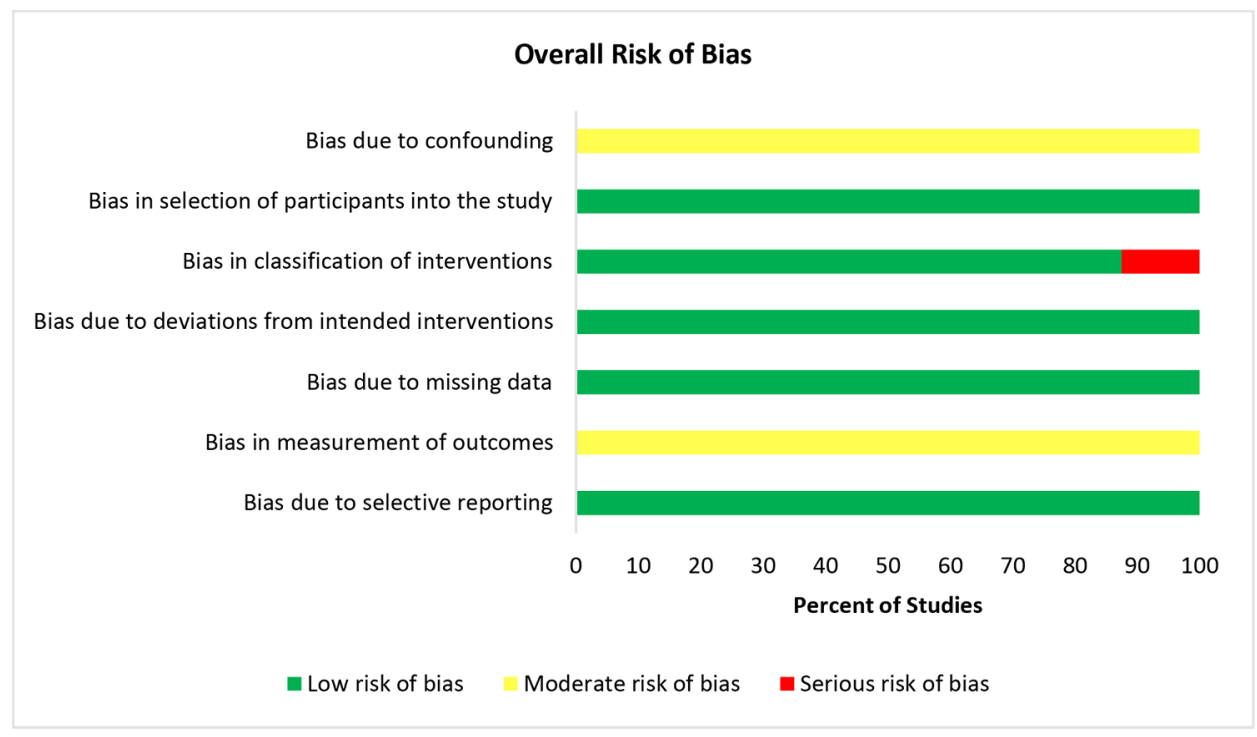

Figure 2 Risk of bias graph. Risk of bias is presented as a percentage across all included studies (green: low risk; yellow: moderate; red: serious). Each row represents a different section of the Cochrane risk of bias assessment tool. If all studies scored the same score for any given section, then that entire row will appear as one colour. 
Systematic review

\begin{tabular}{lcccl}
\hline Table 4 & Bicipital groove pain & & & \\
\hline Study & ABT & OBT & Total & P value \\
\hline Duchman et $a l^{15}$ & $2 / 20(10.0)$ & $5 / 25(20.0)$ & $7 / 45(15.6)$ & 0.36 \\
\hline Forsythe et $a l^{14}$ & $14 / 37(37.8)$ & $22 / 38(57.9)$ & $36 / 75(48.0)$ & 0.08 \\
Gombera et al & $8 / 23(34.8)$ & $10 / 23(43.5)$ & $18 / 46(39.1)$ & 0.55 \\
Tu et al ${ }^{8}$ & $3 / 55(5.5)$ & $0 / 62(0)$ & $3 / 117(2.6)$ & 0.06 \\
Werner et al ${ }^{9 *}$ & $3 / 32(9.4)$ & $3 / 50(6.0)$ & $6 / 82(7.3)$ & 0.57 \\
Yi et al ${ }^{11}$ & $2 / 34(5.8)$ & $0 / 32(0)$ & $2 / 66(3.0)$ & 0.16 \\
Yi et al ${ }^{10}$ & $3 / 36(8.3)$ & $0 / 35(0)$ & $3 / 71(4.2)$ & 0.08 \\
\hline
\end{tabular}

Cases are reported as number of patients with positive pain/total number of patients (\%) at latest follow-up.

${ }^{*}$ Only a fraction of the Werner et al ${ }^{9}$ cohort was included at latest follow-up.

$A B T$, arthroscopic suprapectoral biceps tenodesis; $\mathrm{OBT}$, open subpectoral biceps tenodesis.

reported using the SANE Score, none of which found significant differences in scores at latest follow-up between groups (table 5). Similarly, there were no significant differences in preoperative scores between groups in any of the reported outcomes $(p>0.05)$.

\section{Functional outcomes}

Range of motion

All studies ${ }^{7-11} 14-16$ performed physical examinations of ROM in both the operative and contralateral extremities using a goniometer. Multiple studies found no significant differences between groups in regard to range of shoulder forward flexion, ${ }^{7-11} 1416$ shoulder abduction ${ }^{7-11} 14-16$ or internal/external rotation ${ }^{7-11} 15$ at latest follow-up, except for in one study, ${ }^{15}$ in which OBT patients demonstrated a significantly increased range of shoulder forward flexion when directly compared with ABT patients at latest follow-up $\left(177.8^{\circ} \pm 9.3^{\circ}\right.$ vs $171.3^{\circ} \pm 11.7^{\circ}$ respectively, $\mathrm{p}=0.049)$.

\section{Strength testing}

Five studies ${ }^{79}$ 14-16 used a dynamometer to evaluate strength of both the operative and contralateral extremities postoperatively. All values were compared with strength of the contralateral extremity. Multiple studies found no significant differences between groups in regard to strength with elbow flexion, ${ }^{79}$ 14-16 forearm supination $^{715}$ or shoulder abduction ${ }^{715}$ at latest follow-up $(\mathrm{p}>0.05)$.

\section{Complications}

All studies ${ }^{7-11}$ 14-16 reported on postoperative complications, including wound infection, cosmetic deformity, postoperative stiffness, neural or vascular injury, deep venous thromboses, loss of proximal fixation and need for reoperation. No studies found a significant difference in complication rates between groups, except for in two studies, ${ }^{89}$ in which patients undergoing ABT experienced significantly more postoperative stiffness when compared with OBT patients $(\mathrm{p}<0.05)$.

\section{DISCUSSION}

Arthroscopic suprapectoral and open subpectoral biceps tenodesis are two surgical procedures commonly performed for treatment of various pathologies of the LHBT, including tendinosis, tenosynovitis, complete or partial rupture, subluxation/dislocation or SLAP tears. ${ }^{24}$ Common complications of these procedures include postoperative stiffness, acute bicipital groove pain,

Table 5 Patient-reported outcome scores

\begin{tabular}{|c|c|c|c|c|c|}
\hline \multirow[b]{2}{*}{ Study } & \multicolumn{2}{|l|}{$A B T$} & \multicolumn{2}{|l|}{ OBT } & \multirow[b]{2}{*}{$P$ value } \\
\hline & Preoperative & Postoperative & Preoperative & Postoperative & \\
\hline \multicolumn{6}{|l|}{ ASES } \\
\hline Duchman et al ${ }^{15}$ & NR & $79.6 \pm 22.3$ & NR & $82.3 \pm 21.1$ & 0.68 \\
\hline Forsythe et al ${ }^{14}$ & $42.2 \pm 15.4$ & $85.9 \pm 15.4$ & $44.2 \pm 21.4$ & $85.6 \pm 17.2$ & 0.73 \\
\hline Gombera et al ${ }^{7}$ & NR & $88.9 \pm 3.4$ & NR & $92.3 \pm 3.2$ & 0.42 \\
\hline Green et $a l^{16}$ & NR & $91.4 \pm 13.9$ & NR & $90.6 \pm 11.4$ & 0.69 \\
\hline Tu et $a l^{8}$ & $49.5 \pm 11.1$ & $88.5 \pm 3.4$ & $52.9 \pm 8.2$ & $89.1 \pm 4.0$ & $>0.05$ \\
\hline Werner et $a l^{9 *}$ & NR & $90.1 \pm 13.6$ & NR & $88.4 \pm 10.0$ & 0.74 \\
\hline Yi et $a l^{11}$ & $43.3 \pm 6.9$ & $84.7 \pm 5.5$ & $40.6 \pm 6.6$ & $86.0 \pm 6.2$ & $>0.05$ \\
\hline Yi et $a l^{10}$ & $41.7 \pm 5.3$ & $87.7 \pm 5.3$ & $42.8 \pm 4.7$ & $86.1 \pm 7.6$ & 0.32 \\
\hline \multicolumn{6}{|l|}{ VAS } \\
\hline Green et $a l^{16}$ & NR & $0.9 \pm 1.8$ & NR & $0.7 \pm 1.1$ & 0.74 \\
\hline Tu et $a l^{8}$ & $4.9 \pm 1.5$ & $1.2 \pm 1.4$ & $5.0 \pm 1.1$ & $1.0 \pm 0.7$ & $>0.05$ \\
\hline Yi et $a l^{11}$ & $7.6 \pm 1.0$ & $2.4 \pm 1.0$ & $7.5 \pm 1.0$ & $2.3 \pm 1.0$ & $>0.05$ \\
\hline Yi et $a l^{10}$ & $7.5 \pm 1.0$ & $2.1 \pm 0.9$ & $7.6 \pm 0.7$ & $2.0 \pm 0.8$ & 0.67 \\
\hline \multicolumn{6}{|l|}{ Constant-Murley } \\
\hline Duchman et $a l^{15}$ & NR & $86.7 \pm 11.1$ & NR & $88.0 \pm 19.7$ & 0.77 \\
\hline Tu et $a l^{8}$ & $52.1 \pm 10.5$ & $90.4 \pm 3.1$ & $53.8 \pm 7.2$ & $90.7 \pm 4.3$ & $>0.05$ \\
\hline Werner et $a l^{9 *}$ & NR & $90.7 \pm 10.4$ & NR & $91.8 \pm 6.6$ & 0.76 \\
\hline Yi et $a l^{11}$ & $49.1 \pm 7.5$ & $89.1 \pm 5.4$ & $51.6 \pm 9.7$ & $88.5 \pm 4.6$ & 0.58 \\
\hline \multicolumn{6}{|l|}{ SANE } \\
\hline Duchman et al ${ }^{15}$ & NR & $88.8 \pm 16.1$ & $N R$ & $88.9 \pm 18.0$ & 0.98 \\
\hline Forsythe et al ${ }^{14}$ & $32.7 \pm 20.5$ & $80.8 \pm 22.3$ & $34.5 \pm 21.0$ & $83.0 \pm 19.1$ & 0.86 \\
\hline Werner et $a l^{9 *}$ & NR & $87.4 \pm 13.9$ & NR & $86.8 \pm 10.7$ & 0.90 \\
\hline
\end{tabular}

Scores are reported as a mean \pm SD at latest follow-up.

*Only a fraction of the Werner et $a l^{9}$ cohort was included at latest follow-up. The 'p-value' column represents a comparison of postoperative scores between groups. ABT, arthroscopic suprapectoral biceps tenodesis; ASES, American Shoulder and Elbow Surgeon; NR, not reported; OBT, open subpectoral biceps tenodesis; SANE, Single Assessment Numeric Evaluation; VAS, visual analogue scale. 
and in rare cases, implant failure. Previous studies comparing these two techniques have identified a higher risk of postoperative stiffness and limited ROM in patients undergoing ABT compared with OBT, which was consistent with the findings of this review. ${ }^{91516}$

The results of this systematic review suggest that patients undergoing OBT may experience a decreased incidence of postoperative stiffness. However, no differences in the rate of treatment failure, bicipital groove pain, strength or PROs should be expected when compared with ABT patients. Patients in both groups demonstrated improvements from preoperatively to postoperatively in terms of Constant-Murley Scores, VAS for pain, SANE and ASES scores. Furthermore, in terms of functional performance (strength and ROM), only one study found a significant difference between groups, in which OBT patients demonstrated a significantly increased range of shoulder forward flexion when compared with ABT patients at latest follow-up. However, in accordance with previous studies on the same topic, we identified an occasional increase in postoperative stiffness in patients undergoing ABT. It is also important to note that in the one study that did find significantly decreased forward flexion in ABT patients, ABT patients were significantly older and had significantly more concomitant RCR when compared with the OBT group, which could both contribute to this finding.

The relationship between postoperative stiffness and the arthroscopic suprapectoral tenodesis technique is likely multifaceted. In ABT, a more extensive bursal resection in the subdeltoid space is required to appropriately locate the biceps tendon and identify tenodesis location, which may result in increased tissue disruption and further contribute to the formation of subdeltoid adhesions. Other factors inherent to an arthroscopic suprapectoral technique such as increased fluid extravasation, increased risk of bleeding in the bicipital sheath region or possible overtensioning of the biceps tendon may also result in sensations of stiffness postoperatively. Previous studies have reported that $\mathrm{ABT}$ patients may experience persistent biceps tendinopathy if a proximal tenodesis site leaves a portion of the tendon within the bicipital groove. ${ }^{25-27}$ Intra-articular findings of biceps tenosynovitis extending distally into the bicipital groove suggest an inflammatory component within the groove and may in part explain the feelings of postoperative stiffness in patients undergoing $\mathrm{ABT}^{28}$

The strengths of this study include a comprehensive systematic review performed by two independent reviewers. The limitations of this study should also be noted. In particular, only one of the included studies was of level I evidence. ABT and OBT techniques were not identical across all studies, not all studies reported on the same functional outcomes and not all studies used the same PROs. Due to the moderate heterogeneity present in techniques and outcomes reported between studies, a metaanalysis could not be performed. In addition, follow-up times were short term to midterm and highly variable, ranging from 9 to 50 months. Finally, this study did not stratify outcomes based on indication (biceps tendinosis, partial/complete proximal biceps tendon rupture, SLAP tears, etc).

\section{CONCLUSION}

Patients undergoing ABT and OBT can be expected to experience similar improvements in clinical outcomes at latest follow-up without differences treatment failure or functional performance. ABT patients may experience an increased incidence of stiffness in the early postoperative period.

Contributors Conception and design: all authors; administrative support: JH, ST, ECM and JWB; provision of study materials or patients: JH, ST, ECM and JWB; collection and assembly of data: JH, ST, ECM and JWB; data analysis and interpretation: JH, ST, ECMC and JWB; manuscript writing: all authors; final approval of manuscript: all authors.

Funding The authors have not declared a specific grant for this research from any funding agency in the public, commercial or not-for-profit sectors.

Disclaimer There are no competing interests for any author. There was no funding required for this project. There was no ethical approval required for this project. There are no acknowledgements for this project. All data were available to all authors for the entirety of this project.

Competing interests None declared.

Patient consent for publication Not required.

Provenance and peer review Not commissioned; externally peer reviewed.

Data availability statement All data relevant to the study are included in the article or uploaded as supplementary information. n/a.

\section{REFERENCES}

1 Castricini R, Familiari F, De Gori M, et al. Tenodesis is not superior to tenotomy in the treatment of the long head of biceps tendon lesions. Knee Surg Sports Traumatol Arthrosc 2018;26:169-75.

2 Wilk KE, Hooks TR. The painful long head of the biceps brachii: nonoperative treatment approaches. Clin Sports Med 2016;35:75-92.

3 Chen C-H, Hsu K-Y, Chen W-J, et al. Incidence and severity of biceps long head tendon lesion in patients with complete rotator cuff tears. J Trauma 2005:58:1189-93.

4 Murthi AM, Vosburgh CL, Neviaser TJ. The incidence of pathologic changes of the long head of the biceps tendon. J Shoulder Elbow Surg 2000;9:382-5.

5 Sethi N, Wright R, Yamaguchi K. Disorders of the long head of the biceps tendon. J Shoulder Elbow Surg 1999;8:644-54

6 Hsu AR, Ghodadra NS, Provencher MT, et al. Biceps tenotomy versus tenodesis: a review of clinical outcomes and biomechanical results. I Shoulder Elbow Surg 2011;20:326-32

7 Gombera MM, Kahlenberg CA, Nair R, et al. All-arthroscopic suprapectoral versus open subpectoral tenodesis of the long head of the biceps brachii. Am J Sports Med 2015;43:1077-83.

8 Tu J, Xu B, Guo R. Open subpectoral vs. arthroscopic proximal biceps tenodesis: a comparison study of clinical outcomes. Exp Ther Med 2020;19:428-34.

9 Werner BC, Pehlivan HC, Hart JM, et al. Increased incidence of postoperative stiffness after arthroscopic compared with open biceps tenodesis. Arthroscopy 2014;30:1075-84

10 Yi G, Yang J, Zhang L, et al. Small-Incision open distal subpectoral vs. arthroscopic proximal biceps tenodesis for biceps long head tendon lesions with repair of rotator cuff tears. Exp Ther Med 2020;19:861-70

11 Yi Y, Lee J-M, Kwon SH, et al. Arthroscopic proximal versus open subpectoral biceps tenodesis with arthroscopic repair of small- or medium-sized rotator cuff tears. Knee Surg Sports Traumatol Arthrosc 2016;24:3772-8.

12 Werner BC, Burrus MT, Miller MD, et al. Tenodesis of the long head of the biceps: a review of indications, techniques, and outcomes. JBJS Rev 2014;2:01874474.

13 Higgins JP, Green S, eds. Cochrane Handbook for systematic reviews of intervention. The Cochrane Collaboration, 2011. www.handbook.cochrane.org

14 Forsythe B, Zuke WA, Agarwalla A, et al. Arthroscopic Suprapectoral and open subpectoral biceps Tenodeses produce similar outcomes: a randomized prospective analysis. Arthroscopy 2020;36:23-32.

15 Duchman KR, DeMik DE, Uribe B, et al. Open versus arthroscopic biceps tenodesis: a comparison of functional outcomes. lowa Orthop J 2016;36:79-87.

16 Green JM, Getelman MH, Snyder SJ, et al. All-arthroscopic suprapectoral versus open subpectoral tenodesis of the long head of the biceps brachii without the use of interference screws. Arthroscopy 2017;33:19-25

17 Sterne JA, Hernán MA, Reeves BC, et al. ROBINS-l: a tool for assessing risk of bias in non-randomised studies of interventions. BMJ 2016;355:i4919.

18 McHugh ML. Interrater reliability: the kappa statistic. Biochem Med 2012;22:276-82

19 Richards RR, An KN, Bigliani LU, et al. A standardized method for the assessment of shoulder function. J Shoulder Elbow Surg 1994:3:347-52.

20 Constant CR, Murley AH. A clinical method of functional assessment of the shoulder. Clin Orthop Relat Res 1987;214:160???164-4.

21 Williams GN, Gangel TJ, Arciero RA, et al. Comparison of the single assessment numeric evaluation method and two shoulder rating scales. outcomes measures after shoulder surgery. Am J Sports Med 1999;27:214-21.

22 Werner BC, Evans CL, Holzgrefe RE, et al. Arthroscopic suprapectoral and open subpectoral biceps tenodesis: a comparison of minimum 2-year clinical outcomes. Am J Sports Med 2014;42:2583-90.

23 Coleman BD, Khan KM, Maffulli N, et al. Studies of surgical outcome after patellar tendinopathy: clinical significance of methodological deficiencies and guidelines for future studies. Victorian Institute of sport tendon Study Group. Scand I Med Sci Sports 2000;10:2-11. 


\section{Systematic review}

24 Creech MJ, Yeung M, Denkers M, et al. Surgical indications for long head biceps tenodesis: a systematic review. Knee Surg Sports Traumatol Arthrosc 2016;24:2156-66.

25 Johannsen AM, Macalena JA, Carson EW, et al. Anatomic and radiographic comparison of arthroscopic suprapectoral and open subpectoral biceps tenodesis sites. Am J Sports Med 2013;41:2919-24.
26 Lutton DM, Gruson Kl, Harrison AK, et al. Where to tenodese the biceps: proximal or distal? Clin Orthop Relat Res 2011:469:1050-5.

27 Sanders B, Lavery KP, Pennington S, et al. Clinical success of biceps tenodesis with and without release of the transverse humeral ligament. J Shoulder Elbow Surg 2012;21:66-71.

28 DePALMA AF. Bicipital tenosynovitis. Surg Clin North Am 1953:1693-702. 